\title{
A Privacy-Preserving Roaming Authentication Scheme for Ubiquitous Networks
}

\author{
You-sheng Zhou \\ College of Computer Science and Technology, \\ Chongqing University of Posts and Telecommunications, Chongqing 400065, CHINA \\ College of Computer Science, \\ Chongqing University, \\ Chongqing 400044, CHINA
}

\author{
Jun-feng Zhou \\ College of Computer Science and Technology, \\ Chongqing University of Posts and Telecommunications, \\ Chongqing 400065, CHINA
}

\author{
Feng Wang \\ College of Mathematical Sciences, \\ Dezhou University, \\ Dezhou 253023, CHINA
}

\begin{abstract}
A privacy-preserving roaming authentication scheme (PPRAS) for ubiquitous networks is proposed, in which a remote mobile user can obtain the service offered by a foreign agent after being authenticated. In order to protect the mobile user's privacy, the user presents an anonymous identity to the foreign agent with the assistance of his or her home agent to complete the authentication. After that, the user and the foreign agent can establish a session key using the semi-group property of Chebyshev polynomial. In this way, huge burden of key management is avoided. Furthermore, the user can update the login password and the session key between itself and the foreign agent if necessary. The correctness is proved using BAN logic, and the performance comparison against the existing schemes is given as well.
\end{abstract}

Keywords—roaming authentication; anonymous; chaotic maps; key agreement

\section{INTRODUCTION}

High-speed development of mobile internet has a profound influence on people's daily life. The mobile user wishes to share something or get some resources via mobile devices anytime anywhere and it should not be an issue when he or she locates in the range of the home network provider. However, when a mobile user moves to the region of a foreign network, how does he or she access the foreign network. Undoubtedly, as shown in Fig. 1., the ubiquitous networks should be equipped with authentication and session key establishment before it permits the user to access the Internet provided by itself.

Many authentication and key establishment protocols for mobile networks [1-7] have been proposed in recent years. In 2009 Chang et al. [1] proposed an efficient authentication protocol for mobile devices, which uses one-way hash functions and exclusive-or operation to reduce computation, and they claimed that their scheme can achieve perfect forward secrecy. However, their protocol cannot protect user's privacy since plaintext of real identities are used during the authentication. Later, Chang et al. [2] proposed another enhanced authentication scheme, which uses a random number and one-way hash functions to protect the user's identity, while the scheme cannot prevent insider attack as a malicious inner user can get the real identity at ease. Li et al. [8] proposed an efficient mobile networks authentication scheme, which can protect mobile users' privacy, while it is vulnerable to the manin-the-middle attacks. Shin et al. [9] and Wen et al.[10] proposed anonymous authentication schemes for mobile networks respectively, while Shin et al.'s [9] scheme cannot resist to the man-in-the-middle attacks, and Wen et al.'s [10] scheme will reveal the user's real identity. In 2014, Xie et al. [11] proposed a mobile roaming authentication protocol and claimed this scheme can protect users' privacy; however, its efficiency is not desirable. Mao et al. [12] proposed an anonymous authentication for global mobility networks in the same year. Recently, Farash et al. [13] proposed a light weight authentication scheme for roaming ubiquitous networks, while it is vulnerable to the replay attacks.

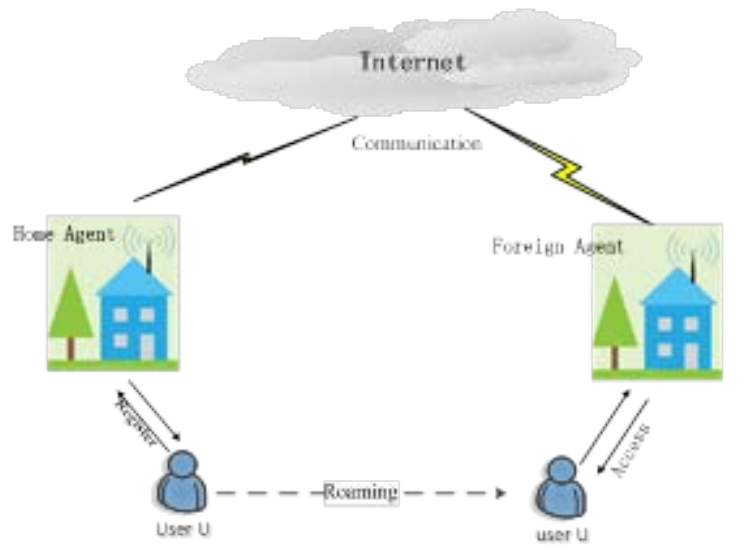

Fig. 1. The scenario of roaming authentication

To improve the security issues, some protocols [14-16] use smart card to authenticate and establish session key. In 2010, 
Li et al. [15] proposed an efficient authentication protocol using smart card to make user be anonymous, which enhances the security with untraceablility property. Recently, much work on Chebyshev chaotic map based authentication with smart card [17-21] have been done. Juang et al. [22] proposed an authenticated key agreement using smart card, which is privacy-preserving and time-synchronization free. However, in 2009, Sun et al. [23] pointed out that Juang et al.'s [22] scheme suffers inability of the password-changing operation and the session-key problem, hence they proposed an improved authentication protocol using smart card. In 2013, Guo et al. [21] proposed a password-authenticated using smart card. In 2015, Lin et al. [24] proposed an improved chaotic maps based authentication protocol using smart card.

As the popularity of mobile network-enabled devices, people are fond of dealing all work on those devices. However, the private information, for example user identification, may be illegally intercepted and then tracked by the potential attackers. However, the existing schemes either fail to provide privacy preserving or incur huge key management, since traditional symmetric or asymmetric encryption is employed for the handshake message. To address mobile users‘ privacy effectively, a privacy preserving roaming authentication and key agreement (PPRAS) is proposed in this paper. In PPRAS, the smart card together with chaotic maps is employed to improve efficiency and simplify the session key agreement and key management. In the proposed scheme, the foreign agent can authenticate the mobile user without knowing the user's real identity, then they can agree the shared session key and the temporary identification.

The rest of the article is organized as follows, some related basics are briefly reviewed in section II. The concrete construction of PPRAS is illustrated in section III. Analysis and comparison are presented in section IV. The paper is concluded in the last section.

\section{PREMILARIES}

A brief introduction of the Chebyshev maps and some related basics are given in this section.

\section{A. Chebyshev Chaotic Maps}

Definition 1: Let $n$ be an integer, $x \in[-1,1]$, an $n$-order Chebyshev polynomial map $T_{n}(x):[-1,1] \rightarrow[-1,1]$ is defined as follows:

$$
T_{n}(x)=\cos (n * \cos (x))
$$

According the definition, the recursive form of Chebyshev polynomial map can be produced as follows

$$
\begin{aligned}
& T_{n}(x)=2 * x * T_{n-2}(x)-T_{n-2}(x), n \geq 2, \\
& \text { where } T_{0}(x)=1, T_{2}(x)=x, n \geq 2 .
\end{aligned}
$$

The Chebyshev polynomial map follows the following two properties
1) Semi-group property

$$
\begin{aligned}
T_{r}\left(T_{s}(x)\right) & =\cos \left(r^{*} \cos ^{-1}\left(s^{*} \cos ^{-1}(x)\right)\right) \\
= & \cos \left(r^{*} s^{*} \cos ^{-1}(x)\right) \\
= & T_{s r}(x)=T_{s}\left(T_{r}(x)\right)
\end{aligned}
$$

where $r, s$ are two integers, $x \in[-1,1]$.

\section{2) Chaos property}

When $n>1$, a $n$-degree Chebyshev polynomial map $T_{n}(x):[-1,1] \rightarrow[-1,1]$ has the constant measure $f^{*}(x)=1 /\left(p \sqrt{1-x^{2}}\right)$ and positive Lyapunov exponent $\lambda=\ln n>0$.

\section{B. The Extended Chebyshev Chaotic Maps}

According to the periodicity of $y=\cos (x)$, there exist multiple $x$ associated with the same $y$ to make the equation hold. Zhang [25] proved that the Chebyshev polynomial map still keeps the semi-group property over the interval $(-\infty, \infty)$, and proposed the concept of the extended Chebyshev chaotic maps as follows.

$$
\left.T_{n}(x)=\left(2 x T_{n-1}(x)\right)-T_{n-2}(x)\right) \bmod P,
$$

where $n \geq 2, x \in[-1,1]$, and $P$ is a big prime number. Furthermore, the following equation holds as well:

$$
T_{r}\left(T_{s}(x)\right)=T_{s r}(x)=T_{s}\left(T_{r}(x)\right) \bmod P
$$

Definition 2: Discrete Logarithm Problem (DL)

Given any two big integer $x, y$, find an integer $s$ to satisfy the equation $T_{s}(x) \equiv y$.

Definition 3: Decisional Diffie-Hellman Problem (DDH)

Given $T_{r}(x), T_{s}(x), T_{u}(x)$, where $r, s, u$ are unknown, determine the equation $T_{s r}(x)=T_{u}(x) \bmod P$ holds or not.

\section{CONSTRUCTION OF PPRAS}

In this section, the detailed construction of PPRAS is presented. For convenience, the descriptions of some symbols to be used are listed in TABLE I.

In PPRS, there exist three entities: the mobile user $M U$, the home agent $H A$ and the foreign agent $F A$. When $M U$ moves to $F A$ 's network, $F A$ needs to authenticate $M U$ before giving him the permission to access the network. To finish the authentication, $F A$ needs the assistance of $H A$ to verify whether $\mathrm{MU}$ is an authorized user or not. If not, the authentication process will be terminated. The proposed scheme consists four stages: registration phase, authentication phase including session key establishment, session key update and login password update phase.

During the initialization, FA shares a session key with $H A$, which is securely stored locally. The authentication is launched by $M U$, and then proceeds as the following interactive steps. 
TABLE I. DESCRIPTION OF SYMBOLS

\begin{tabular}{|c|c|}
\hline Symbol & Description \\
\hline$I D_{i}$ & identification of communication entity $i$ \\
\hline SID & temporary identification of mobile user(MU) \\
\hline$T_{n}(x)$ & Chebyshev polynomial with degree $n$ \\
\hline$T_{s}$ & $T_{s}(x)$ \\
\hline$T_{M U}, T_{F A}$ & $T_{r_{M U}}(x), T_{r_{F A}}(x)$ \\
\hline$X$ & the initial value of chaotic map \\
\hline$S$ & private key of the home agent \\
\hline$P$ & a big prime number \\
\hline$x_{i}, r_{i}$ & random numbers chosen by users \\
\hline$K_{M U}$ & session key shared between $M U, F A$ and $H A$ \\
\hline$k_{H F}$ & the shared key between $F A$ and $H A$ \\
\hline$E(.) / D()$. & symmetric encryption/decryption algorithm \\
\hline$t_{M U}, t_{F A}$ & timestamp \\
\hline$\Delta T$ & threshold of interval \\
\hline$H()$. & a secure one-way hash function \\
\hline$\oplus$ & XOR operation \\
\hline$P W_{M U}$ & password of mobile user \\
\hline$T_{H}$ & running time for hash operation \\
\hline$T_{E}$ & running time for encryption operation \\
\hline
\end{tabular}

\begin{tabular}{|l|l|}
\hline$T_{D}$ & running time for decryption operation \\
\hline$T_{C}$ & running time for chaotic map operation \\
\hline$T_{M}$ & running time for modular exponential operation \\
\hline
\end{tabular}

\section{A. Registration Phase}

A mobile user $M U$ registers himself in his or her home agent $H A$ using the following steps,

1) HA chooses two random numbers $x$, s and a big prime number $P$, then computes $T_{s}=T_{s}(x) \bmod P$, and publishes $\left(X, T_{\mathrm{s}}, P\right)$.

2) $M U$ chooses his $P W_{M U}$ and a random number $\lambda$, then computes $H=h\left(P W_{M U}, \lambda\right)$, then sends $\left\{I D_{M U}, H\right\}$ to $H A$ via a secure channel.

3) $H A$ checks the validity of $I D_{M U}$ and $H\left(P W_{M U}, \lambda\right)$ using $H\left(H\left(P W_{M U}, \lambda\right) \| I D_{M U}\right)$. If yes, computes the message $I M=h\left(I D_{M U}\|s\| t_{\text {reg }}\right)$ which respect the identity of $M U$, with his secret key $s$ and the timestamp $t_{\text {reg }}$, then store the parameters $\left\{I D_{M U}, H, I M, X, T_{s}, I D_{H A}, H(),. E(\right.$.$) ,$ $T_{n}(),$.$P \} into a smart card and send it to \mathrm{MU}$, where $T_{n}($. is a Chebyshev polynomial with degree $n$ among them. Otherwise, MU fails to register in the system.

\section{B. Authentication and Key Establishment Phase}

$M U$ and $F A$ can complete the authentication and establishment by following the steps shown in Fig. 2.

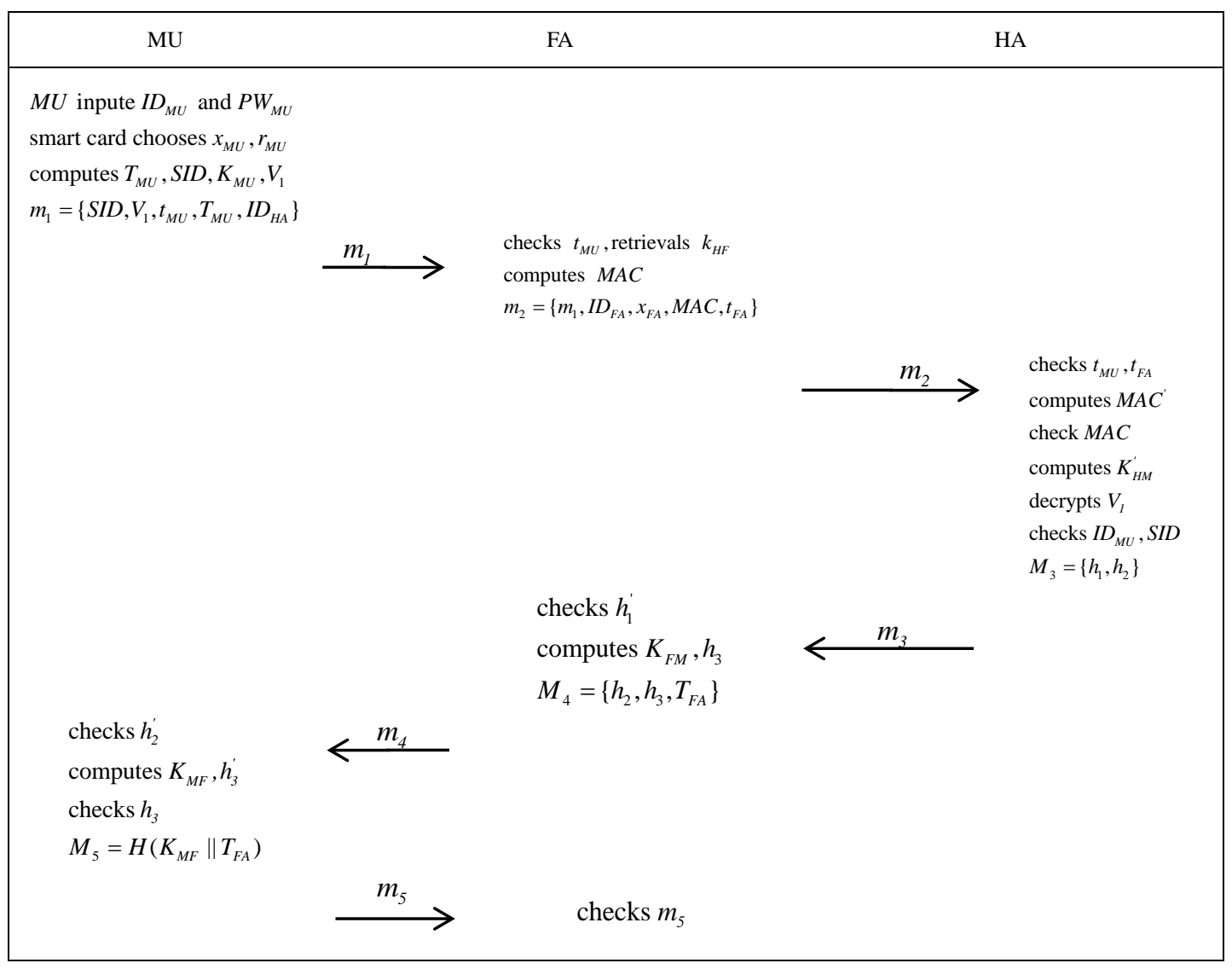

Fig. 2. The process of authenticating and key establishing 
- $\quad M U \rightarrow F A: m_{1}=\left\{S I D, V_{1}, T_{M U}, t_{M U}, I D_{H A}\right\}$

$M U$ first inputs his real identity $I D_{M U}$ and password $P W_{M U}$ into the smart card, then the smart card (SC) make the decision that allowing $M U$ to login or not by computing $H^{\prime}=h\left(P W_{M U}, \lambda\right)$ and checking validity of $I D_{M U}$ and $H^{\prime}$ ? $=H$. If yes, $S C$ chooses two random numbers: $x_{M U}$, $r_{M U}$, then computes $T_{M U}=T_{r_{M U}}(x) \bmod P$, $K_{M H}=T_{r_{M U}}\left(T_{s}\right) \bmod P$ and $S I D=I D_{M U} \oplus H\left(x_{M U}\right)$, where SID denotes the temporary identification of $M U$, and $\mathrm{K}_{\mathrm{MH}}$ denotes the shared session key between $M U$ and $H A$. After that, $S C$ encrypts $I D_{M U}, I D_{H A}, I M, X_{M U}$ and the current timestamp $\mathrm{t}_{\mathrm{MU}}$ using $K_{M H}$, that is $V_{1}=E_{K_{M H}}\left(I D_{M U}\left\|I D_{H A}\right\| I M\left\|x_{M U}\right\| t_{M U}\right)$. Next, $M U$ sends $m_{1}=\left\{S I D, V_{1}, T_{M U}, t_{M U}, I D_{H A}\right\}$ to $F A$.

- $F A \rightarrow H A: m_{2}=\left\{m_{1}, I D_{F A}, x_{F A}, M A C, t_{F A}\right\}$

Upon receiving $m_{1}$ from $M U, F A$ firstly checks $\left|t_{F A}-t_{M U}\right|<\Delta T$ holds or not, where $t_{F A}$ is the current time of $F A, \Delta T$ denotes the permissible threshold of time interval. If yes, stores SID temporarily firstly, and then searches the shared session key $k_{H F}$ between $F A$ and $H A$ using $I D_{H A}$. Next, computes the message authentication code $M A C$ :

$M A C=h\left(I D_{F A}\left\|V_{1}\right\| x_{F A}\left\|t_{F A}\right\| k_{H F}\right)$, where $x_{F A}$ is a random number chosen by $F A$ temporarily. At last, sends the message $m_{2}=\left\{m_{1}, I D_{F A}, x_{F A}, M A C, t_{F A}\right\}$ to $H A$.

- $H A \rightarrow F A: m_{3}=\left\{h_{1}, h_{2}\right\}$

After receiving $m_{2}$ from $F A, H A$ firstly checks $\left|T-\mathrm{t}_{\mathrm{FA}}\right|<\Delta \mathrm{T}$ , $\left|T-\mathrm{t}_{\mathrm{MU}}\right|<\Delta \mathrm{T}$ holds or not, where $T$ denotes the timestamp of $H A, \Delta T$ denotes the permissible threshold of time interval.

If these two equation hold, $H A$ confirms $I D_{F A}$ and $S I D$ as follows:

Step 1. Uses $I D_{F A}$ retrievals the shared session key $k_{H F}$ between $H A$ and $F A$, then computes $M A C^{\prime}=h\left(I D_{F A}\left\|V_{1}\right\| x_{F A}\left\|t_{F A}\right\| k_{H F}\right)$ and checks whether $M A C^{\prime}=M A C$ holds or not.

Step 2. If yes, $H A$ confirms the identity $I D_{F A}$ from $F A$, then computes $K_{H M}=T_{s}\left(T_{M U}\right) \operatorname{modP}$ to decrypt $V_{1}$, and checks whether $t_{M U}, I D_{H A}$ in $V_{1}$ are all equal to the plaintext $t_{M U}, I D_{H A}$ in message $m_{1}$. if yes, uses the decrypted $I D_{M U}$ to retrieval his database to check whether $t_{M U}>t_{\text {reg }}$. If holds, computes $I M^{\prime}=h\left(I D_{M U}\|s\| t_{\text {reg }}\right)$ and $S I D^{\prime}=I D_{M U} \oplus H\left(x_{M U}\right)$, then checks if $I M$ ? $=I M^{\prime}$ and $S I D ?=S I D^{\prime}$. If they all hold, confirms the anonymous identity SID is valid.

Step 3. Computes the message $m_{3}=\left\{h_{1}=h\left(S I D\left\|x_{F A}\right\| k_{H F} \| h_{2}\right), h_{2}=h\left(I M\left\|x_{M U}\right\| k_{H M}\right)\right\}$ , then sends it to $F A$.

- $F A \rightarrow M U: m_{4}=\left\{h_{2}, h_{3}, T_{F A}\right\}$

After receiving $\mathrm{m}_{3}$ from $H A, F A$ firstly computes $h_{1}^{\prime}=h\left(S I D\left\|x_{F A}\right\| k_{H F} \| h_{2}\right)$, then checks if $h_{1}^{\prime}=h_{1}$. If yes, confirms the temporary identification SID of $M U$ is valid. After that, $F A$ chooses a random number $r_{F A}$, then computes $T_{F A}=T_{r_{F A}}(x) \bmod P \quad, \quad K_{F M}=T_{F A}\left(T_{M U}\right) \bmod P \quad$ and $h_{3}=h\left(S I D\left\|k_{F M}\right\| h_{2} \| T_{F A}\right)$, where $K_{F M}$ is the session key between $F A$ and $M U$, then sends $m_{4}=\left\{h_{2}, h_{3}, T_{F A}\right\}$ to $M U$.

- $\quad M U \rightarrow F A: m_{5}=h\left(K_{M F} \| T_{F A}\right)$

After receiving $m_{4}$ from $F A, \quad M U$ firstly computes $h_{2}^{\prime}=h\left(I M\left\|x_{M U}\right\| K_{H M}\right)$, checks whether $h_{2}=h_{2}^{\prime}$ holds or not. If yes, $M U$ confirms $F A$ is authenticated, then computes the following values: $K_{M F}=T_{M U}\left(T_{F A}(x)\right) \bmod P$ and $h_{3}^{\prime}=h\left(S I D\left\|k_{M F}\right\| h_{2}^{\prime} \| T_{F A}\right)$, where $K_{M F}$ is the session key between $M U$ and $F A$, then checks if $h_{3}^{\prime} ?=h_{3}$. If yes, establishes the session key $K_{M F}$, then $M U$ computes the message $m_{5}=h\left(k_{M F} \| T_{F A}\right)$ and sends it to $F A$.

- Upon receiving $m_{5}$ from $M U$, $F A$ firstly computes $m_{5}^{\prime}=h\left(k_{F M} \| T_{F A}\right)$, then check if $M_{5}^{\prime} ?=M_{5}$. If holds, completes establishing the session key.

\section{Session Key Update Phase}

In order to ensure the security, it is necessary for $M U$ to periodically update the session key established previously between himself and FA. MU follows the following steps to update his or her session key in the $i^{\text {th }}$ time:

Step 1. MU firstly chooses a number $t_{i}$ randomly, computes $\quad T_{v M U}=T_{t_{i}}(x) \bmod P \quad$ and $m_{i}=\left\{E_{K_{M F_{i}}}\left(S I D, T_{v M U}, t_{v M U}, C h\right), S I D, t_{v M U}\right\}$, then sends the message $m_{i}$ to $F A$, where $S I D$ is the anonymous identity of $M U$ when he establishes the session key $k_{M F_{i}}$ between himself and $F A$ at the previous time, $t_{v M U}$ is current timestamp, $C h$ is the flag to denotes update query.

Step 2. Upon receiving the message $m_{1}$ from $M U$, firstly checks $\left|T_{i}-t_{v M U}\right|<\Delta T$ holds or not, where $T_{i}$ is the current time of $F A, \Delta T$ denotes the permissible threshold of time interval. If yes, FA uses SID to get the $i^{\text {th }}$ session key $K_{F M_{i}}$ to decrypt $m_{i}$ and check whether SID is equal to the plaintext SID . 
If yes, $F A$ chooses a random number $r_{i+1}$, and computes

$$
T_{F A_{i+1}}=T_{r_{i+1}}(x) \bmod P
$$

$k_{F M_{i+1}}=T_{F A_{i+1}}\left(T_{v M U}(x)\right) \bmod P$, where $k_{F M_{i+1}}$ is the current session key. Then FA sends the message $m_{i+1}=\left\{E_{K_{F M_{i}}}\left(h\left(K_{F M_{i+1}} \| K_{F M_{i}}\right), T_{F A_{i+1}}, I D_{F A}\right), I D_{F A}\right\}$ to MU

Step 3. After receiving $m_{i+1}$ from $F A, M U$ firstly uses the previous session key $k_{M F_{i}}$ to decrypt $m_{2}$, computes the new session key $k_{M F_{i+1}}=T_{v M U}\left(T_{F A_{i+1}}(x)\right) \bmod P$ and $h^{\prime}\left(K_{F M_{i+1}} \| K_{F M_{i}}\right)$, then checks if $h^{\prime}\left(K_{F M_{i+1}} \| K_{F M_{i}}\right)=h\left(K_{F M_{i+1}} \| K_{F M_{i}}\right)$ holds or not. If yes, completes session key update.

\section{Login Password Update Phase}

It is necessary for $M U$ to update his or her login password dynamically to prevent someone else who knows his or her password from doing some impersonation attacks. The update of login password can be finished as follows:

Step 1. MU puts his or her smart card into the reader, then inputs his or her real identity $I D_{M U}$ and the password $P W_{M U}$, then the smart card can make the decision that allowing $M U$ to login or not by computing $H^{\prime}=h\left(P W_{M U}, \lambda\right)$ and checking validity of $I D_{M U}$ and $H^{\prime} ?=H$. If yes, $M U$ sends update request.

Step 2. When the smart card receives the request, it asks $M U$ to input the new password $P W_{M U}^{\prime}$, and a random number $t$ ' if necessary, then the smart card computes $H^{\prime}=h\left(P W_{M U}^{\prime}, t^{\prime}\right)$ and updates it.

\section{ANALYSIS OF PPRAS}

\section{A. Correctness Analysis}

The Burrows-Abadi-Needham (i.e. BAN) logic [27] is useful to identify some possible weakness in the security protocols, especially for the authentication protocol, so the BAN logic is used to analyze the correctness of PPRAS. Some notations are listed in TABLE II.

TABLE II. NOTATIONS FOR BAN LOGIC

\begin{tabular}{ll}
\hline Notation & Description \\
\hline$A \mid \equiv X$ & $A$ trusts $\mathrm{X}$, or $A$ believes $\mathrm{X}$ \\
$A \triangleleft \mathrm{X}$ & $A$ sees $\mathrm{X}$, or $A$ holds $\mathrm{X}$ \\
$A \mid \sim \mathrm{X}$ & $A$ has said $\mathrm{X}$ \\
$A \mid \Rightarrow \mathrm{X}$ & $A$ completely controls over $\mathrm{X}$ \\
Rule 1 & Rule 2 comes from Rule 1 \\
\hline Rule 2 & \\
$A \stackrel{\mathrm{X}}{\longrightarrow} B$ & $X$ is a secret key or information between $A$ and $B$ \\
$\{\mathrm{X}\}_{K}$ & $\mathrm{X}$ is encrypted by the key $K$ \\
\hline
\end{tabular}

1) Idealization

According to the rules of the BAN logic, the first step is to idealize the authentication phases of PPRAS as follows:

a) $M U \rightarrow F A:$

$m_{1}=\left\{S I D,\left\{I D_{M U}\left\|I D_{H A}\right\| I M\left\|x_{M U}\right\| t_{M U}\right\}_{M U \stackrel{K_{M H} \rightarrow H A}{ }}\right.$,

$$
\left.T_{M U}, t_{M U}\right\}
$$

b) $F A \rightarrow H A$ :

$$
m_{2}=\left\{m_{1}, h\left(I D_{F A}\left\|V_{1}\right\| x_{F A}\left\|t_{F A}\right\| F A \stackrel{K_{F H}}{\longleftrightarrow} H A\right), t_{F A}\right\}
$$

c) $H A \rightarrow F A$

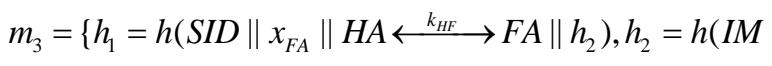$$
\left.\left.\left\|x_{M U}\right\| H A \stackrel{k_{H M}}{\longleftrightarrow} M U\right)\right\}
$$

d) $\mathrm{FA} \rightarrow \mathrm{MU}$

$$
m_{4}=\left\{h_{2}, h_{3}=h\left(S I D\left\|F A \stackrel{k_{F M}}{\longleftrightarrow} M U\right\| h_{2} \| T_{F A}\right), T_{F A}\right\}
$$

$$
\text { e) } M U \rightarrow F A: m_{5}=h\left(M U \stackrel{k_{M F}}{\longrightarrow} F A \| T_{F A}\right)
$$

2) Assumptions

In PPRAS, there exist three entities: the mobile user $(M U)$, the foreign agent (FA) and the home agent (HA). Each entity has his or her possessions and abilities. The initial assumptions are descripted as follows:

For $M U$ :

$$
\begin{aligned}
& \text { A1. } M U \triangleleft I D_{M U} \\
& \text { A2. } M U \mid \equiv S I D \\
& \text { A3. } M U \mid \equiv I D_{H A} \\
& \text { A4. } M U \equiv r_{M U} \\
& \text { A5. } M U \mid \equiv M U \stackrel{K_{M H}}{\longleftrightarrow} H A
\end{aligned}
$$

A1: $M U$ believes his or her own identity.

A2: $M U$ believes his or her own pseudonym SID .

A3: As $M U$ registers himself in his home agent $H A$ to be a legitimate user, so he believes $H A$ 's identity $I D_{H A}$.

A4: $M U$ believes the number $x_{M U}$ chosen by himself.

A5: $M U$ believes the session key $K_{M H}$ between himself and $H A$, because $K_{M H}$ is computed using the Chebyshev polynomials with $H A$ 's public parameter $T_{H A}$ and $T_{M U}$.

For $H A$ :

$$
\begin{aligned}
& \text { A6: } H A \triangleleft I D_{H A} \\
& \text { A7: } H A \mid \equiv \#\left(t_{M U}\right) \\
& \text { A8: } H A \mid \equiv \#\left(t_{F A}\right) \\
& \text { A9: } H A \mid \Rightarrow s \\
& \text { A10: } H A \mid \equiv H A \stackrel{k_{\mathrm{HF}}}{\longleftrightarrow} F A
\end{aligned}
$$

A6: HA holds his own identity.

A7: $H A$ believes $t_{M U}$ is fresh, and has never received it before so that he can authenticate $M U$. 
A8: $H A$ believes $t_{F A}$ is fresh, and has never received it before so that he can authenticate $F A$.

A9: As $s$ is $H A$ 's secret key, so $H A$ completely controls over his secret key $s$.

A10: $H A$ believes the key shared between $H A$ and FA before authenticating.

For FA:

$$
\begin{aligned}
& \text { A11: } F A \triangleleft I D_{F A} \\
& \text { A12: } F A \triangleleft I D_{H A} \\
& \text { A13: } F A \mid \equiv \#\left(t_{M U}\right) \\
& \text { A14: FA } \equiv F A \stackrel{k_{\mathrm{HF}}}{\longrightarrow} H A \\
& \text { A15: FA } \equiv r_{F A}
\end{aligned}
$$

A11: FA holds his own identity.

A12: FA needs to authenticate $M U$ with the help of $H A$, so he needs to hold $H A$ 's identity $I D_{H A}$.

A13: FA believes $t_{M U}$ is fresh so that he will be able to finish the next operation.

A14: FA believes the session key $K_{F H}$ between himself and $H A$, because $K_{F H}$ is computed using Chebyshev polynomials with $H A$ 's public parameter $T_{H A}$ and the value $T_{F A}$ computed by FA himself.

\section{3) Goals}

According to the proposed scheme, $M U$ and FA want to establish a session key with the help of $H A$, so our proposed scheme needs to achieve the following goals:

$$
\begin{aligned}
& \mathrm{G} 1: H A \mid \equiv S I D \\
& \mathrm{G} 2: H A \mid \equiv I D_{F A} \\
& \mathrm{G} 3: F A|\equiv H A| \equiv S I D \\
& \mathrm{G} 4: M U|\equiv H A| \equiv I D_{F A} \\
& \mathrm{G} 5: M U \mid \equiv M U \stackrel{K_{M F}}{\longleftrightarrow} F A \\
& \mathrm{G6}: F A \mid \equiv F \stackrel{K_{F M}}{\longrightarrow} M U
\end{aligned}
$$

G1: $H A$ believes the anonymous identity of $M U$.

G2: HA believes FA's identity.

G3:FA believes that $H A$ has verified MU's anonymous identity SID.G4: $M U$ believes that $H A$ believes $F A$ is a legitimate agent.

G5: $M U$ believes the session key between himself and FA, that is $M U$ has already successfully generated the session key with $F A$.

G6: FA believes the session key between himself and $M U$, that is $F A$ has already successfully generated the session key with $M U$.

$M U$ wants to establish a session key with FA without leaking his identify, he needs an anonymous identify which used to be authenticated by $H A$, and $H A$ must believe FA's identify to enable $M U$ to communicate with FA. After they finish the process of generating the session key, FA and $M U$ must believe the authenticated peer holds the common session key.

4) Verification

In this section, the BAN logic is employed to check whether PPRAS is correct or not. The primary steps are shown as follows:

Theorem 1. HA believes the anonymous identity of $M U$ and the identity of FA.

Proof :

$$
\begin{aligned}
& \mathrm{V} 1: \frac{H A \triangleleft m_{2}}{\frac{H A \triangleleft h\left(I D_{F A}\left\|V_{1}\right\| x_{F A}\left\|t_{F A}\right\| k\right), H A \mid \equiv H \stackrel{k_{\mathrm{HF}}}{H} F A}{H A F\left|\sim I D_{F A}, H A\right| \equiv \#\left(h\left(I D_{F A}\left\|V_{1}\right\| x_{F A}\left\|t_{F A}\right\| k_{\mathrm{HF}}\right)\right)}} \\
& \text { V2: }
\end{aligned}
$$

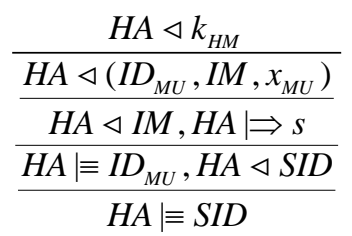

According to the assumption A7 and A8, HA believes the message $m_{1}$ and $m_{2}$ are fresh, and he has never received them before, applying the seeing rule:

$$
\frac{A \triangleleft(x, y)}{A \triangleleft x},
$$

$H A$ holds $h\left(I D_{F A}\left\|V_{1}\right\| x_{F A}\left\|t_{F A}\right\| k_{H F}\right)$, with the assumption A10, applying the message-mean rule: $\frac{P \mid \equiv P \stackrel{k}{\longleftrightarrow} Q, P \triangleleft\{x\}_{k}}{P|\equiv Q| \sim x}$,

$H A$ believes that $F A$ has said $I D_{F A}$, applying fresh rule: $\frac{A \mid \equiv \#(x, y)}{A \mid \equiv \# x}$,

$H A$ believes $h\left(I D_{F A}\left\|V_{1}\right\| x_{F A}\left\|t_{F A}\right\| k_{H F}\right)$ is fresh, applying nonce-verification rule:

$$
\frac{P|\equiv \#(x), P| \equiv Q \mid \sim x}{P|\equiv Q| \equiv x},
$$

$H A$ believes that FA believes $I D_{F A}$, so $H A$ believes $I D_{F A}$.

After verifying the correction of MAC, $H A$ believes the session key $k_{H F}$, so he believes the identity of $F A$, and also believes the message $m_{1}$ has not been tampered. Then $H A$ computes $K_{H M}$ to decrypt $V_{1}$ in message $m_{1}$, applying the seen rule:

$$
\frac{A \triangleleft(x, y)}{A \triangleleft x},
$$

$H A$ can get $I D_{M U}, I M$ and $x_{M U}$. According to the assumption A9, $H A$ believes the real identify of $M U$, then $H A$ 
can verify the anonymous identity of $M U$ with the received value $S I D$ in message $m_{1}$.

With the proof above, it can be found that $H A$ believes the anonymous identity ( $S I D$ ) of $M U$ and the identity ( $I D_{F A}$ ) of FA.

Theorem 2. FA believes that $H A$ has verified $M U$ 's anonymous identity SID.

Proof:

$$
\text { V3: } \quad \frac{F A \triangleleft m_{3}}{F A \triangleleft h_{1}, F A \mid \equiv A \stackrel{k_{\mathrm{HF}}}{\mathrm{F}} H A}
$$

After $F A$ receives the message $m_{3}$ from $H A$, applying the seen rules:

$$
\frac{A \triangleleft(x, y)}{A \triangleleft x},
$$

$F A$ receives the value $h_{1}$ in message $m_{3}$. According to the assumption A14, we know that $H A$ has verify the anonymous identity $S I D$, so $F A$ believes that $H A$ believes the anonymous identity $S I D$ of $M U$ after he verify the message $h_{1}$ in the received message $m_{3}$.

Above all, FA believes that $H A$ has verified MU's anonymous identity SID.

Theorem 3. $M U$ believes that $H A$ believes $F A$ is a legitimate agent.

Proof:

$$
\text { V4 }: \frac{\mathrm{MU} \triangleleft m_{4}}{\frac{M U \triangleleft h_{2}, M U \mid M U \stackrel{k_{H M}}{M} H A}{M U\left|\mathrm{~h}_{2}, M U\right| \equiv r_{M U}}}
$$

After $M U$ receives the message $m_{4}$ from $F A$, applying the seeing rules:

$$
\frac{A \triangleleft(x, y)}{A \triangleleft x},
$$

$M U$ receives the value $h_{2}$ in message $m_{4}$. According to the assumption A5, MU believes the value $h_{2}$, and also believes $M U$ believes that $H A$ believes FA's identity after he or she verifies $h_{2}$ under the assumption A4. So $M U$ believes that $H A$ believes $F A$ is a legitimate agent.

Theorem 4. $M U$ believes the session key between himself and $F A$, that is $M U$ has already generated the session key with FA.

Proof:

$$
\text { V5: } \quad \frac{M U \triangleleft m_{4}}{\frac{M U \triangleleft h_{3}, M U \mid \equiv\left(S I D, h_{2}\right)}{M U \mid \equiv h_{3}, M U \triangleleft K_{M F}}}
$$

After $M U$ receives the message $m_{4}$ from $F A$, applying the seeing rules:

$$
\frac{A \triangleleft(x, y)}{A \triangleleft x},
$$

$M U$ receives the value $h_{3}$ in message $m_{4}$. According to the assumption A2 and the theorem 3, MU believes $h_{3}$.

After $M U$ verifies $h_{2}$, he or she computes the session key $K_{M F}$ between $M U$ and $F A$, so $M U$ holds $K_{M F}$, according to the proof above that MU believes $h_{3}, M U$ can verify the key $K_{M F}$ is right with $h_{3}$, that is $M U$ believes the session key between himself and FA.

Theorem 5. FA believes the session key between himself and $M U$, that is FA has already generated the session key with MU.

Proof:

$$
\text { V6: } \quad \frac{F A \mid \equiv r_{F A}}{F A \equiv T_{F M}, F A \triangleleft m_{5}}
$$

After $F A$ receives the message $m_{5}$ from $M U$, according to the assumption A15, applying the belief rules:

$$
\frac{A \mid \equiv x}{A \mid \equiv(x, y)},
$$

FA believes $T_{F M}$, as $F A$ holds the message $m_{5}$, so $F A$ believes the session key $K_{F M}$ between $F A$ and $M U$.

\section{B. Performance Analysis}

The performance evaluation of the existing protocols [9-13] and PPRAS will be discussed in this section. The overall resultes are listed in TABLE III.

TABLE III. COMPARISON ON PERFORMANCE

\begin{tabular}{ccc}
\hline & Client & Server \\
\hline Farash et.al.'s[13] & $6 T_{H}$ & $6 T_{H}+2 T_{E}+2 T_{D}$ \\
Mao et al.'s[12] & $8 T_{H}+2 T_{E}$ & $4 T_{H}+2 T_{E}+4 T_{D}$ \\
Xue et al.'s[11] & $4 T_{H}+T_{E}+T_{D}$ & $3 T_{H}+2 T_{E}+3 T_{D}$ \\
Shin et al.'s[9] & $4 T_{H}$ & $7 T_{H}+2 T_{E}+2 T_{D}$ \\
Wen et al.'s[10] & $4 T_{H}+T_{M}$ & $10 T_{H}+3 T_{M}$ \\
PPRAS & $4 T_{H}+T_{E}+2 T_{C}$ & $9 T_{H}+T_{D}+2 T_{C}$ \\
\hline
\end{tabular}

Since the authentication is a series of synchronized processes, the total computational cost of the client and server during the authentication and key agreement should be investigated. As the cost of XOR operation and module addition are rather cheap, these two operations are not included in the comparison, and only symmetric encryption/decryption operation, chaotic map operation, hash operation and modular exponential operation are evaluated. As shown in TABLE III, the computational cost of client in $[9,10,13]$ is much cheap than PPRAS, however, as discussed previously, the scheme in 
$[9,12]$ cannot resist to the man-in-the-middle attacks, and the scheme in [10] cannot preserve the user's privacy. However, the efficiency of [11] is not desirable. The scheme in [13] is vulnerable to the replay attacks. Furthermore, the schemes in [9-13] will inevitably incur huge key management for the symmetric and public key encryption. Although no explicit advantage of performance for PPRAS cannot be found in TABLE III, the underlying featured chaotic map based encryption for handshake message would save much more computation and storage cost.

\section{Security Analysis}

In this section, the security analysis and performance comparison are illustrated.

1) User Anonymity: The user who wants to authenticate others should provide its real identifies to the trusted three party in the 3PAKE [26] protocol. If the user transfers authentication messages including his identity in plaintext via an insecure channel, an attacker can identify the user by intercepting and analyzing the message, this is not a a desirable scheme for authentication. In PPRAS, the real identity of mobile user is encrypted with the session key computed using Chebyshev polynomial. Even if the adversary got the ciphertext, he or she still faces the difficulty of solving DL hard problem if he or she want to compute the decryption key. Since the temporary identification of MU is generated with the XOR operation on the random number and real identity, it is infeasible in polyninomial time to guess the right identity since the space of identity is big enough. Therefore, FA can get nothing about the user's real identity and the privacy of the useris preserved well.

2) Resistance to The Man-in-The-Middle Attack: Suppose there exists an active attacker over the communication channel, who attempts to intercept and tamper the messages transferred via this channel to carry out the man-in-the-middle attack. If the attacker wants to tamper $m_{1}$, he or she needs to tamper $V_{1}$ in message $m_{1}$ produced by symmetric encryption with the session key, which is computed with the Chebyshev polynomials. However the attacker will face the difficulty of solving the $D L$ problem. As for the messages $m_{2}, m_{3}, m_{4}$, $m_{5}$ generated with the secure one-way hash functions, if the attacker wants to tamper them, he or she will face the difficulty of breaking the secure one-way hash functions according to the definition of the protocol. Above all, PPRAS is secure enough to counter the man-in-the-middle attack.

3) Forward Secrecy: In PPRAS, the forward secrecy means that even if an adversary has obtained the current session key and the password of $M U$, he or she cannot deduce the previous used session key. The agreement of the session key $K_{M F}$ ( or $K_{F M}$ ) between MU and FA is based on the random number $x_{M U}$ and $x_{F A}$, and even $M U$ does not know $x_{M U}$ which is chosen dynamically by the smart card, so the adversary can get nothing about $K_{M F}$ ( or $K_{F M}$ ), that is , the proposed scheme achieves forward secrecy.

4) Backward Secrecy: The backward secrecy of PPRAS refers to the adversary cannot successfully fulfil authentication and session key agreement with the password of $M U$ and all previous used session key together with the current session key. However, all the messages are produced by the smart card and transferred in anonymous way, thus he or she cannot generate a valid message without possesing this smart card according to the protocol, even if he or she is given $P W_{M U}$. So PPRAS achieves the backward secrecy.

5) Resistance to Password Guessing Attack: This attack means that an attacker attemps to deduce the password of the user with interception and analysis over the transferred messages. In PPRAS, however, there does not exist user's password in all these messages, and the attacker can get nothing about user's passwordThus, the proposed scheme can resist to password guessing attack.

6) Resistance to The Replay Attack: According to the construction of the presented protocol, all the transffered messages among $M U, F A$ and $H A$ combine the timestamp $t_{F A}, t_{M U}$ to provide freshness. What's more, the paramters ( $\left.x_{M U}, r_{M U}\right)$ and $\left(x_{F A}, r_{F A}\right)$ are chosen randomly to ensure freshness at the beginning of every authentication session. So the adversary can not replay those messages.

Finally, the overall security comparison of PPRAS and the existing similar schemes are listed in TABLE IV. As shown in the table, only PPRAS can achieve all the security features.

TABLE IV. COMPARISON ON SECURITY

\begin{tabular}{|c|c|c|c|c|c|c|}
\hline $\begin{array}{l}\text { Security } \\
\text { Features }\end{array}$ & $\begin{array}{c}\text { Farash } \\
\text { et.al.'s[13] }\end{array}$ & $\begin{array}{l}\text { Mao et } \\
\text { al.'s[12] }\end{array}$ & $\begin{array}{l}\text { Li et } \\
\text { al.'s[8] }\end{array}$ & $\begin{array}{l}\text { Shin et } \\
\text { al.'s.[9] }\end{array}$ & $\begin{array}{l}\text { Wen et } \\
\text { al.'s[10] }\end{array}$ & PPRAS \\
\hline Forward Secrecy & $\sqrt{ }$ & $\sqrt{ }$ & $\sqrt{ }$ & & $\sqrt{ }$ & $\sqrt{ }$ \\
\hline $\begin{array}{l}\text { Backward } \\
\text { Secrecy }\end{array}$ & $\checkmark$ & $\sqrt{ }$ & $\sqrt{ }$ & $\sqrt{ }$ & ， & $\sqrt{ }$ \\
\hline Anti-replay attack & ' & $\sqrt{ }$ & $\sqrt{ }$ & $\sqrt{ }$ & $\sqrt{ }$ & $\sqrt{ }$ \\
\hline Anti-MIM attack & , & , & & & $\sqrt{ }$ & $\sqrt{ }$ \\
\hline User Anonymity & $\sqrt{ }$ & $\sqrt{ }$ & $\sqrt{ }$ & ' & $\sqrt{ }$ & $\sqrt{ }$ \\
\hline $\begin{array}{l}\text { Anti-guessing } \\
\text { attack }\end{array}$ & $\sqrt{ }$ & . & 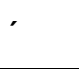 & , & , & $\checkmark$ \\
\hline
\end{tabular}

\section{CONCLUSIONS}

Roaming authentication is essential to the ubiquitous networks, and a lot of efforts have been done to better the security and performance in authentication. However, the existing authentication protocols cannot avoid the huge burden of key agreement and management for authentication which comes from the encryption and poses a barrier to apply it to the multi-user situations. Thus a novel roaming authentication scheme based on Chebyshev chaotic map with user anonymity is proposed in this paper. With the advantage of semi-group property of Chebyshev polynomial, the entities involved in the authentication can agree the session key at low cost, and no additional key management is needed. Meanwhile, the foreign agent can authenticate the user without knowing his real identity, which achieves privacy preserving for the user.

\section{ACKNOWLEDGEMENT}

This work was jointly supported by the National Social Science Foundation of China (no. 14CTQ026), the National Natural Science Foundation of China (no. 61472464), the 
Chongqing Research Program of Application Foundation and Advanced Technology (no. cstc-2014jcyjA40028, no. cstc2013jcyjA40017), the Natural Science Foundation of Shandong Province, China (no. ZR2015FL024).

\section{REFERENCES}

[1] C. C. Chang, S. Y. Lin, J. H.Yang," Efficient user authentication and key establishment protocols with perfect forward secrecy for mobile devices," Computer and Information Technology, Ninth IEEE International Conference on. IEEE,Xiamenpp.131-135, 2009.

[2] C. C.Chang, C. Y. Lee, Y. C. Chiu, " Enhanced authentication scheme with anonymity for roaming service in global mobility networks," Computer Communications, vo.32, no.4, pp.611-618, 2009.

[3] D. He, S. Chan, "A secure and light weight user authentication scheme with anonymity for the global mobility network," Network-Based Information Systems (NBiS), 2010 13th International Conference on. IEEE, Takayama, Gifu, pp.305-312, 2010.

[4] C. Chen, D. He, S. Chan, et al, "Light weight and provably secure user authentication with anonymity for the global mobility network," International Journal of Communication Systems, vol.24, no.3, pp.347362, 2011.

[5] T. Zhou, J. Xu, "Provable secure authentication protocol with anonymity for roaming service in global mobility networks," Computer Networks, vol.55, no.1, pp.205-213, 2011.

[6] J. Xu,W. T. Zhu, D. G. Feng, "An efficient mutual authentication and key agreement protocol preserving user anonymity in mobile networks," Computer Communications, vol.34, no.3, pp.319-325, 2011.

[7] J. Lu, J. Zhou, "On the Security of an Efficient Mobile Authentication Scheme for Wireless Networks," IACR Cryptology ePrint Archive, pp.126, 2010.

[8] H. Li,Y. Yang, L. Pang, "An efficient authentication protocol with user anonymity for mobile networks," Wireless Communications and Networking Conference (WCNC), 2013 IEEE, Shanghai, pp.1842-1847.

[9] S. Shin, H. Yeh, K. Kim, "An efficient secure authentication scheme with user anonymity for roaming user in ubiquitous networks," Peer-toPeer Networking and Applications, pp.1-10, 2013.

[10] F. Wen, W. Susilo, G. Yang, "A secure and effective anonymous user authentication scheme for roaming service in global mobility networks," Wireless personal communications, 2013, pp.993-1004.

[11] Q. Xie, D. Hong, M. Bao, N. Dong and D. S. Wong,., "PrivacyPreserving Mobile Roaming Authentication with Security Proof in Global Mobility Networks," International Journal of Distributed Sensor Networks, 2014.

[12] K. Mao, J. Chen, J. Liu and D. Tong, "An anonymous roaming authentication scheme in global mobility networks," Communications Security Conference (CSC 2014), IET, Beijing, pp.1-4, 2014.
[13] M.S.Farash, S.A.Chaudhry, M.Heydari,et al, "A lightweight anonymous authentication scheme for consumer roaming in ubiquitous networks with provable security," International Journal of Communication Systems, 2015.

[14] D. He, M. Ma, Y. Zhang, C. Chen and J. Bu "A strong user authentication scheme with smart cards for wireless communications," Computer Communications, vol.34, no.3, pp.367-374, 2011.

[15] X. Li, W. Qiu, D. Zheng, K. Chen and J. Li, “Anonymity enhancement on robust and efficient password-authenticated key agreement using smart cards," Industrial Electronics, IEEE Transactions on, vol.57, no.2, pp.793-800, 2010.

[16] D. Wang, P. Wang, J. Liu, "Improved privacy-preserving authentication scheme for roaming service in mobile networks,"Wireless Communications and Networking Conference (WCNC), 2014 IEEE Istanbul, pp.3136-3141, 2014.

[17] C. C. Lee, "A simple key agreement scheme based on chaotic maps for VSAT satellite communications," International Journal of Satellite Communications and Networking, vol.31, no.4, pp.177-186, 2013.

[18] K. Chain, W. C. Kuo, "A new digital signature scheme based on chaotic maps,” Nonlinear Dynamics, vol.74, no.4, pp.1003-1012, 2013.

[19] M. S. Hwang, S. K. Chong, T. Y. Chen, "DoS-resistant ID-based password authentication scheme using smart cards," Journal of Systems and Software, vol.83, no.1, pp.163-172, 2010.

[20] R. Song, "Advanced smart card based password authentication protocol," Computer Standards \& Interfaces, vol.32, no.5, pp.321-325, 2010.

[21] C. Guo, C. C. Chang, "Chaotic maps-based password-authenticated key agreement using smart cards," Communications in Nonlinear Science and Numerical Simulation, vol.18, no.6, pp.1433-1440, 2013.

[22] W.S.Juang, S.T.Chen, H.T. Liaw, "Robust and efficient passwordauthenticated key agreement using smart card,” IEEE Trans Ind Electron vol.55, no.6, pp.2551-2556, 2008.

[23] D. Z. Sun, J. P. Huai, J. Z. Sun, et al, "Improvements of Juang's password-authenticated key agreement scheme using smart cards," Industrial Electronics, IEEE Transactions on, vol.56, no.6, pp.2284-2291, 2009.

[24] H.Y. Lin, "Improved chaotic maps-based password-authenticated key agreement using smart cards," Communications in Nonlinear Science and Numerical Simulation, vol.20, no.2, pp. 482-488, 2015.

[25] L. Zhang, "Cryptanalysis of the public key encryption based on multiple chaotic systems,” Chaos, Solitons \& Fractals, vol.37, no.3, pp.669-674, 2008.

[26] C. T. Li, C. W. Lee, J. J.Shen, "A secure three-party authenticated key exchange protocol based on extended chaotic maps in cloud storage service," Information Networking (ICOIN), 2015 International Conference on. IEEE, Cambodia, pp.31-36, 2015.

[27] M. Burrows, M. Abadi, R. Needham, “A logic of authentication,” ACM Trans Comput Syst, vol.426, no.1871, pp.18-36, 1989. 